\title{
Erratum to: Architectural design of the pelvic floor is consistent with muscle functional subspecialization
}

\author{
Lori J. Tuttle • Olivia T. Nguyen • Mark S. Cook • \\ Marianna Alperin • Sameer B. Shah • Samuel R. Ward • \\ Richard L. Lieber
}

Published online: 29 November 2014

(C) The International Urogynecological Association 2014

\section{Erratum to: Int Urogynecol J DOI 10.1007/s00192-013-2189-5}

We found a mathematical error in sarcomere length $\left(\mathrm{L}_{\mathrm{s}}\right)$ measurements in the original publication. Upon detection of the error, sarcomere lengths were re-measured from the original slide-mounted samples, and also in newly dissected fiber bundles from the same specimens to confirm accuracy. Architectural parameters were then recalculated using corrected $\mathrm{L}_{\mathrm{s}}$ and all statistical analyses were repeated using corrected data. The revised calculations affected the absolute values only and showed that the pelvic floor muscles (PFMs) do not have sarcomeres shorter than other human skeletal muscles. Importantly, there are no changes in the major conclusion of the article regarding the functional subspecialization of the individual PFMs. A table

containing the revised values and the adjusted Figs. 2 and 3 are presented below.

Complete data for coccygeus (C), iliococcygeus (IC), and pubovisceralis (PV) muscles, expressed as means \pm SEM

\begin{tabular}{lllll}
\hline Muscle & Mass $(\mathbf{g})$ & $\mathbf{L}_{\mathbf{f n}}(\mathbf{m m})$ & $\mathbf{L}_{\mathbf{s}}(\boldsymbol{\mu m})$ & $\operatorname{PCSA}\left(\mathbf{c m}^{\mathbf{2}}\right)$ \\
C & $3.91 \pm 0.48$ & $42.95 \pm 3.48$ & $2.52 \pm 0.06$ & $0.87 \pm 0.08$ \\
IC & $4.89 \pm 0.38$ & $57.97 \pm 5.29$ & $2.64 \pm 0.07$ & $0.83 \pm 0.07$ \\
PV & $6.39 \pm 0.98$ & $78.19 \pm 7.39$ & $2.77 \pm 0.12$ & $0.79 \pm 0.08$ \\
P-values* & & & & \\
C vs IC & 0.025 & 0.0061 & 0.89 & 0.53 \\
C vs PV & 0.019 & 0.0002 & 0.1 & 0.71 \\
IC vs PV & 0.16 & 0.0005 & 0.7 & 0.92
\end{tabular}

$\mathrm{L}_{\mathrm{fn}}$, Fiber length normalized to optimal sarcomere length $\left(\mathrm{L}_{\mathrm{s}}\right)$; PCSA, physiological cross-sectional area. ${ }^{*} \mathrm{P}$-values derived from repeatedmeasures ANOVA, followed by Tukey's pairwise comparisons

The online version of the original article can be found at http://dx.doi.org/ 10.1007/s00192-013-2189-5.

\section{J. Tuttle}

Department of Orthopaedic Surgery, University of California San

Diego, San Diego, CA, USA

\section{O. T. Nguyen}

Department of Bioengineering, University of California San Diego, San Diego, CA, USA

\section{S. Cook}

Department of Integrative Biology and Physiology, University of Minnesota, Minneapolis, MN, USA

\section{Alperin}

Department of Reproductive Medicine, Division of Female Pelvic Medicine and Reconstructive Surgery, University of California San Diego, San Diego, CA, USA
S. B. Shah

Departments of Orthopaedic Surgery and Bioengineering, University of California San Diego, San Diego, CA, USA

S. R. Ward

Departments of Radiology, Orthopaedic Surgery and Bioengineering, University of California San Diego, San Diego, CA, USA

R. L. Lieber $(\bowtie)$

Departments of Orthopaedic Surgery and Bioengineering, University of California San Diego, 9500 Gilman Drive, Mail Code 0863, La Jolla, CA 92093, USA

e-mail: rlieber@ucsd.edu

R. L. Lieber

VA San Diego Healthcare System, San Diego, CA, USA 
Fig. 2 Structural and biochemical properties of human pelvic floor muscles. Values are means \pm standard error of the mean (SEM). *Muscle is different from the other two muscles $(\mathrm{P}=0.05)$. PCSA, physiological cross-sectional area; $\mathrm{C}$, coccygeus muscle; IC, iliococcygeus muscle; $\mathrm{PV}$, pubovisceralis muscle
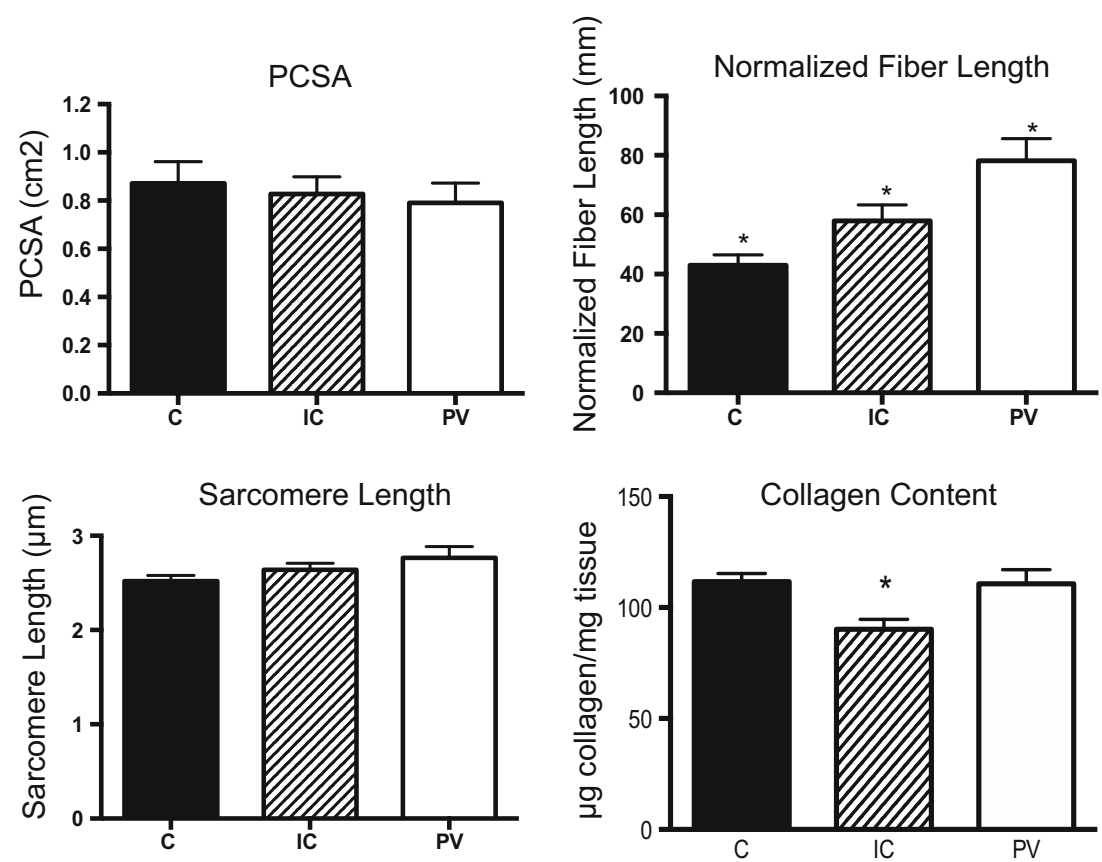

Fig. 3 Normalized fiber length and physiological cross-sectional area (PCSA) for human pelvic floor muscles. Values are means \pm standard error of the mean (SEM)

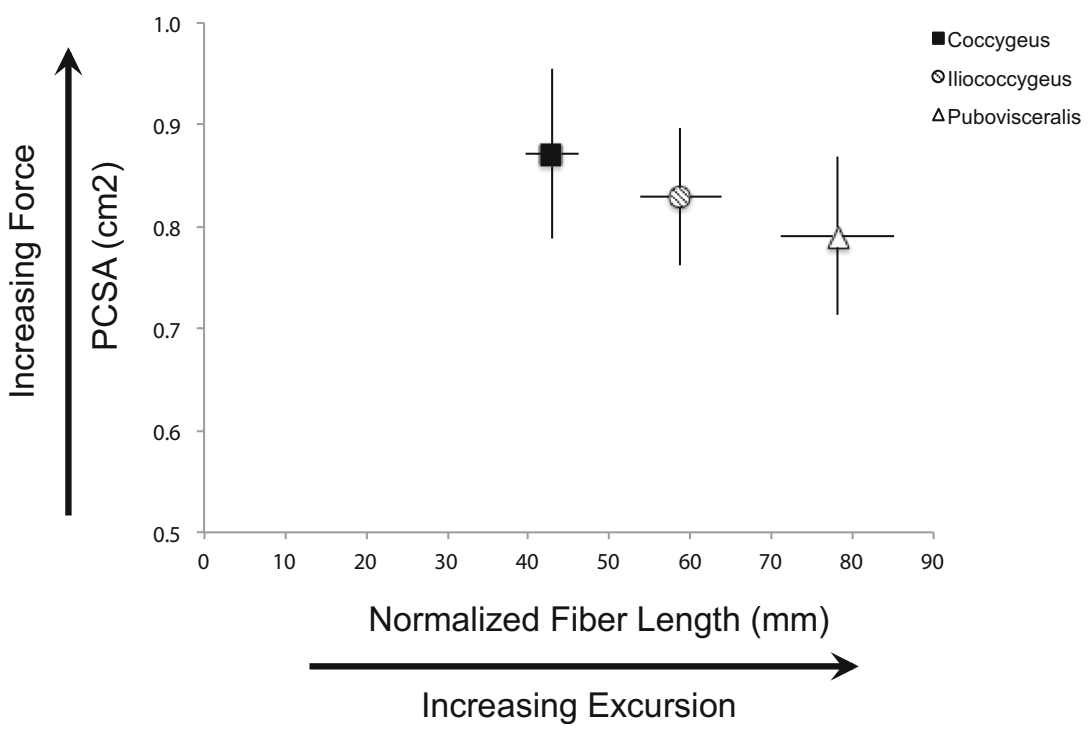

